\title{
圈Sociología
}

Poma, A. (2019). El papel de las emociones en la defensa del medioambiente: Un enfoque sociológico. Revista de Sociología, 34(1), 43-60. doi: 10.5354/0719-529X.2019.54269

\section{El papel de las emociones en la defensa del medioambiente: Un enfoque sociológico}

\section{[The role of emotions in the defense of the environment: A sociological approach]}

\author{
Alice Poma (i)
}

Universidad Autónoma de México

\section{Resumen}

El objetivo del presente artículo es proporcionar conocimiento sobre el papel de las emociones en la defensa del medioambiente a través del análisis de dos conflictos socioambientales en dos de las áreas urbanas más grandes de México: la Ciudad de México y la Zona Metropolitana de Guadalajara. La comparación de dos casos de estudio permite destacar algunos patrones comunes que emergieron del análisis de los datos cualitativos recopilados a través de observación participante, entrevistas en profundidad y grupos focales entre 2015 y 2018. Con base en la literatura sobre movimientos sociales y emociones, se mostrará cómo los apegos al lugar, vinculados con valores biosféricos, al interactuar con los compromisos afectivos y las emociones morales, permiten comprender lo que mueve lo sujetos a defender el medio ambiente.

Palabras clave: emociones, conflictos socioambientales, ciudad, valores.

\begin{abstract}
The aim of this article is to provide knowledge regarding the role of emotions in the defense of the environment through the analysis of two socioenvironmental conflicts in two of the largest urban areas in Mexico: Mexico City and the Metropolitan Zone of Guadalajara. The comparison of two case studies allows us to highlight some common patterns that emerged from the analysis of the qualitative data collected through participant observation, in-depth interviews, and focus groups between 2015 and 2018. Based on literature on social movements and emotions, I will show how place attachments, which are linked with biospheric values, when interacting with affective loyalties and moral emotion, allow to undertand what makes people to defend the environment.
\end{abstract}

Keywords: emotions, environmental conflicts, cities, values. 


\section{INTRODUCCIÓN}

El siglo XXI es el siglo de la crisis ambiental global, causada por el desarrollo industrial y exacerbada por las políticas neoliberales de las últimas décadas, y de la cual el cambio climático es uno de sus síntomas más aterradores. Esta crisis ha generado respuestas en todo el mundo. Entre éstas destacan las resistencias de los pueblos indígenas en defensa de sus territorios experiencias que han llevado a sugerir que existe un "ecologismo de los pobres" (Martínez Alier, 2004)-, que se suma al ecologismo surgido a partir de los años setenta del siglo XX en los países industrializados, y a los conflictos socioambientales (muchas veces identificados de manera despectiva como NIMBY por las siglas en inglés de not in my backyard) que se se dan en áreas rurales como urbanas, en todos los países (Dear, 1992; Poma, 2014).

Solo en México, a principio de 2016 se contaron 420 conflictos sociomabientales (Enciso, 2016), y esto se debe a que "la urbanización y el capitalismo industrial transforman sistemáticamente el paisaje, amenazando el estilo de vida de las personas de una manera que a menudo incentiva la protesta" (Jasper, 1997, p. 94). Sin embargo, las personas que protestan son una minoría, ya que el descontento solo ocasionalmente se convierte en movilización (Jasper, 2014), y por eso es tan importante comprender cómo y por qué la gente se moviliza. Si los movimientos sociales "pueden cambiar nuestro mundo" (Jasper, 2014, p. 14), en el contexto de la crisis ambiental global los conflictos socioambientales son experiencias valiosas no sólo porque frenan la destrucción ambiental, denunciando y oponiéndose a proyectos que amenazan a los territorios y sus habitantes humanos y no humanos, sino también porque en ellos emergen y se fortalecen nuevas narrativas, valores y prácticas proambientales (Poma, 2017, 2019) o, como señala Leff (2004, 2011), una nueva racionalidad ambiental.

La comprensión de los conflictos socioambientales en este artículo se alcanza a través del análisis de dos pequeñas agrupaciones de ciudadanos que se han organizado de manera informal para defender el agua y un bosque en dos de las áreas urbanas más grandes de México: la Ciudad de México y la Zona Metropolitana de Guadalajara (ZMG).

Una relevancia adicional de estos casos reside en la dificultad que presupone defender el medioambiente en las grandes ciudades. $\mathrm{Al}$ ser lugares construidos socialmente como espacios desiguales y antinaturales, en los que tienen prioridad los movimientos de mercancía y dinero, los recursos naturales solo adquieren valor cuando producen beneficios económicos, y la flora y la fauna se convierten a menudo en obstáculos para el desarrollo. En las grandes ciudades, siempre en expansión, se promueven el pendularismo, la movilidad motorizada y el consumo de productos procesados e industriales, que dañan al medioambiente. Los conflictos socioambientales urbanos, se enfrentan así, no solo a los responsables de la destrucción 
ambiental, sino también a la indiferencia o hostilidad de los demás ciudadanos que no cuestionan su estilo de vida.

El objetivo del artículo es proporcionar conocimiento sobre lo que mobiliza a estos sujetos a defender el medioambiente, en contraposición a la indiferencia y/o inacción que caracteriza a la mayoría de los habitantes de las ciudades frente a la devastación ambiental que generan la expansión de las mismas. Para comprender lo anterior, se analizó de qué forma los apegos al lugar (Devine-Wright, 2009; Devine-Wright \& Batel, 2017; Low, 1992), vinculados a los valores biosféricos (Steg, 2016), al interactuar con los compromisos afectivos y las emociones morales (Jasper, 1997, 2012, 2018), influyen en la movilización proambiental. La comparación de dos casos de estudio permite destacar algunos patrones comunes que se pueden observar en el análisis de los datos cualitativos recolectados a través de observación participante, entrevistas en profundidad y grupos focales entre 2015 y 2018. De esta manera se contribuirá a fortalecer la teoría de la acción propuesta por Jasper (2018) acerca del papel de las emociones en la protesta.

En cuanto a la organización del artículo, en el primer apartado se presentarán algunos conceptos empleados en el análisis, para posteriormente describir los casos de estudio y el método de la investigación. El análisis subsecuente incluirá la presentación de un esquema que resume los resultados de la investigación, cuya discusión se verá enriquecida por el conjunto de evidencias como resultado del trabajo de campo.
Emociones y protesta para comprender la defensa del medioambiente

La incorporación de la dimensión emocional para comprender los movimientos sociales ocurrió a partir del giro cultural de este campo de estudio en los años noventa del siglo pasado (Johnston \& Klandermans, 1995). Desde entonces se demostró que el análisis del papel de las emociones contribuye a comprender la mayoría de las dinámicas de los movimientos sociales (e.g., reclutamiento, consolidación, movilización, agotamiento) y a raíz de ello complementar las teorías clásicas (Jasper, 1997). Fue así que emergió la línea de análisis de emociones y protesta en el campo de estudio de los movimientos sociales.

Entre las obras que destacan en esta línea, además de Jasper (1997, 2012, 2014, 2018), la obra colectiva coordinada por Goodwin, Jasper y Polletta (2001), muestra la relevancia de las emociones tanto en el reclutamiento, como en la consolidación y en la disolución de la protesta política, tanto en contextos democráticos como represivos (Goodwin \& Pfaff, 2001). Sucesivamente, Flam y King (2005) ofrecieron una compilación que muestra la conexión entre el nivel micropolítico de los movimientos sociales con el meso- y macropolítico, aspecto que ya había sido analizado en Flam (2000). Además, se evidencian la relevancia de las emociones en la etapa inicial de la movilización y en su continuación; las dinámicas emocionales entre los participantes, con los espectadores y entre diferentes movimientos sociales. 
Gould (2009) ofrece un análisis de ACT UP (AIDS Coalition to Unleash Power) en Estados Unidos, analizando no sólo el papel de diferentes emociones en diferentes etapas del movimiento, sino también el manejo emocional de estas emociones, como la canalización del dolor en rabia, o de la vergüenza en orgullo. En dicho estudio se muestra cómo los activistas consiguieron transformar el dolor experimentado por la pérdida de amigos y compañeros a causa del sida, en rabia contra el estado que fue acusado de no hacer lo suficiente para enfrentar la enfermedad.

La consolidación de esta línea de investigación (para mayores antecedentes véase: Poma \& Gravante, 2017a, 2018) hizo que se reconociera como uno de los nuevos paradigmas del campo de estudio de los movimientos sociales (Diani, 2015), y que se propusiera como una teoría de la acción (Jasper, 2018). La aplicación de esta teoría a los conflictos socioambientales, aún escasa en la academia latinoamericana, ha demostrado su capacidad para comprender dinámicas que otros enfoques, como la ecología política, no abarcan. Poma (2017) por ejemplo, muestra de qué manera la aplicación de la propuesta de Jasper (1997, 2012) permite comprender lo que mueve a los afectados por represas a resistir en contra de estas infraestructuras, y cómo la experiencia de la lucha genera un impacto en sus vidas.

Con la finalidad de mostrar el papel de las emociones en dos conflictos socioambientales, el artículo se apoya en la propuesta de James Jasper (1997, 2018) que organizó las emociones relevantes para la protesta en cinco tipologías
(Jasper 1998, 2012, 2018): (a) impulsos; (b) emociones reflejo; (c) lealdades o compromisos afectivos; y (d) estados de ánimo y emociones morales. Las tipologías se construyeron a partir de algunas características de las emociones, como el grado de procesamiento cognitivo, mayor en las emociones morales; la duración, las emociones reflejo son las más rápidas; y la direccionalidad hacia un objeto, en este caso diferenciando los estados de ánimos de las demás emociones.

Este artículo se centrará en los compromisos afectivos y las emociones morales, como claves de la comprensión de la movilización de los sujetos analizados. Además, se mostrará la relación entre estas emociones y los valores biosféricos "que hacen que las personas se centren en las consecuencias de sus elecciones para la naturaleza y el medioambiente" (Steg, 2016, p. 279), partiendo de la idea de que las respuestas emocionales que alimentan la acción están vinculadas con los valores de los sujetos (Pearlman, 2013; Perlaviciute, Steg, Contzen, Roeser \& Huijts, 2018).

La relación entre valores y emociones es evidente en las emociones morales, que dependen de lo que las personas consideramos justo o injusto (Jasper, 2012). Por ejemplo, un evento o información que un sujeto perciba como una injusticia, le genera indignación. Sin embargo, los valores de las personas también influyen en los vínculos afectivos y las consecuencias emocionales de la protesta, de momento que "actuar moralmente bien [...] puede hacer que las personas se sientan bien consigo mismas" (Steg, 2016, p. 281). Este vínculo entre valores y 
emociones explica los beneficios emocionales de la prefiguración política, pues "si deseamos incorporar la moralidad en las ciencias sociales como un conjunto de motivaciones para la acción, debemos reconocer las emociones involucradas: hacer lo correcto se siente bien" (Jasper, 2018, p. 14).

Un último concepto que es necesario introducir es el de manejo emocional (Hochschild, 1979, 1983, 2008), que es la capacidad de los seres humanos de hacer encajar lo que sentimos y la expresión de nuestras emociones en el contexto social. El manejo emocional, que puede ser consciente o inconsciente, ocurre al seguir o desafiar ciertas reglas del sentir que se construyen cultural y socialmente, y forma parte del aprendizaje de los activistas ambientales (Gravante \& Poma, 2018; Kleres \& Wettergren, 2017). Estos conceptos serán aplicados para mostrar el papel de las emociones en la defensa del medioambiente, en los casos que se presentarán a continuación.

Los casos de estudio en comparación

A pesar de ser dos casos que nunca colaboraron entre sí, y que están ubicados en geografías diferentes, la diferencia más grande de las dos experiencias aún activas es que la lucha del Comité Salvabosque (CS) en el municipio de Zapopan (Jalisco) empezó en mayo de 2005, mientras que la lucha de la Asamblea General de Pueblos, Barrios, Colonias y Pedregales de Coyoacán (en lo sucesivo, AG) surgió en la Alcaldía de Coyoacán (Ciudad de México) en febrero de 2016. Tanto el bosque defendido por el
CS, como el acuífero somero que los integrantes de la AG llaman "el manantial", se encuentran amenazados por desarrollos inmobiliarios, aunque en el caso del CS son fraccionamientos compuestos por casas, y en el caso de la AG un conjunto de 377 departamentos y más de 600 cajones de estacionamiento.

Los dos casos surgieron porque los vecinos se dieron cuenta de que algo no estaba bien en su colonia. En el caso del CS el evento que desencadenó el conflicto fue la tala de trescientos árboles, y en el caso de la AG, el drenaje del agua del acuífero a la calle, bajo responsabilidad de la empresa constructora.

La conformación de los dos grupos también es parecida. El CS está constituido por alrededor de cuarenta vecinos, siendo menos de una decena los que se dedican al comité prácticamente de tiempo completo. En el caso de la AG son aproximadamente sesenta vecinos, siendo poco más de una docena quienes que se turnaban en el plantón (sitting) para las guardias y las actividades cotidianas. En los dos colectivos hay personas de diferentes generaciones, desde niños hasta personas mayores, de los cuales varios tienen vínculos consanguíneos. Este elemento será relevante a la hora de analizar el papel de las emociones recíprocas entre los miembros de las agrupaciones.

Otra característica común a los dos casos de estudio es la relación entre los integrantes de los grupos y el territorio, que se caracteriza por un fuerte apego al lugar local (Low, 1992). En ambos casos, los habitantes de las colonias (i.e., zonas al interior de una ciudad) han forjado sus territorios, contribuyendo a hacerlos vivibles con 
la instalación de servicios básicos como agua potable, electricidad, y pavimentación, de los que carecían. La experiencia relacionada con la transformación del territorio, que supuso esfuerzos y sacrificios, explica el sentimiento de pertenencia al mismo que se evidencia en el fuerte apego al lugar local (Poma, 2019; Poma \& Gravante, 2017b).

En cuanto a la organización, las dos agrupaciones son informales que decidieron colectivamente no formalizarse como asociaciones civiles, al imponer esta figura jurídica una jerarquía entre las agrupaciones que podría distorsionar su forma de ser. Ambas organizaciones toman decisiones en asamblea, y los espacios donde realizan sus actividades son puestos a disposición por sus integrantes o apropiados con acción directa no violenta, como fue la instalación de un plantón por parte de la AG en la Alcadía de Coyoacán, o la ocupación del bosque para actividades de reforestación, limpieza, educación ambiental, entre otras, por parte del CS. Ambas organizaciones son apartidistas y no reciben dinero de ninguna organización gubernamental o no gubernamental, autofinanciándose con actividades autónomas como kermesses, eventos y eventos culturales.

Aun siendo colectivos urbanos -en los que la mayoría de sus miembros nacieron en este contexto y tienen un estilo de vida urbano, y que trabajan, por ejemplo, en los sectores secundario y terciario-, en ambos se puede observar la presencia de culturas indígenas, lo cual es posible dada la vinculación directa de algunas familias con algunos pueblos indígenas.
Una característica que también se considera relevante destacar es el carácter prefigurativo de ambas luchas. En la literatura sobre movimientos sociales, es posible distinguir una tendencia prefigurativa en distintos movimientos sociales (Epstein, 1991; Graeber, 2009; Players, 2010; Yates, 2014). Sin embargo, son escasos los análisis que evidencian el carácter prefigurativo de los conflictos socioambientales. La política prefigurativa tiende a involucrar toda una serie de prácticas alternativas adicionales a las actividades que se desarrollan para defender el medioambiente, como la organización horizontal y antijerárquica, la toma de decisiones por consenso, la acción directa no violenta y la práctica del do it yourself, entre otras. De este modo, la política prefigurativa hace emerger también un proceso de creación de alternativas políticas aquí y ahora (Maeckelbergh, 2011; Players, 2010), sin esperar la intervención del Estado.

La prefiguración política, que se traduce en recrear en la lucha aquí y ahora el mundo al que aspiran los defensores del medioambiente, se puede ver en los grupos analizados tanto en la prevención de incendios, el cuidado de la vegetación y la reforestación que lleva a cabo el CS, así como en la autogestión y creación de espacios comunitarios, como la casa y el vivero donde se reúnen los miembros del CS y el plantón de la AG, y en las actividades culturales como los talleres de arte y música, o la Escuela Popular Piedra y Manantial que realiza la AG antes de su asamblea semanal. 


\section{MÉTODO}

El presente artículo se basa en datos cualitativos producidos a través de la observación participante, entrevistas en profundidad $\mathrm{y}$ grupos focales.

La observación y el seguimiento en redes sociales se focalizaron en observar las acciones colectivas de los dos grupos. Las entrevistas se centraron en la experiencia personal de los integrantes más activos. En total se entrevistaron a ocho miembros del CS y 13 de la AG. En ambos casos, las entrevistas se realizaron en un 50\% a hombres y en un $50 \%$ a mujeres. Se procuró además contar con entrevistas a personas de diferentes generaciones: dos jóvenes de veinte años, dos personas mayores de más de setenta años y los demás adultos entre 30 y 50 .

El uso de las tres técnicas de investigación permitió triangular los datos que cada una proporciona. Las entrevistas en profundidad se centraron en el papel de las emociones, ya que no todas las personas se sienten cómodas y están acostumbradas a expresar su sentir en momentos colectivos. Además, las entrevistas también permiten conocer el contexto de la lucha, los valores de los entrevistados y acceder a informaciones biográficas de éstos, que permiten comprender la dimensión emocional de estas experiencias. La observación participante permite constatar cómo se expresan las emociones y los valores en las prácticas y discursos cotidianos, y la interacción entre actores. Por último, los grupos focales permiten analizar la interacción entre los miembros de los colectivos y discutir con ellos dinámicas emocionales que emergieron con las otras técnicas, además de algunas hipótesis de la investigación. La aplicación de esta técnica fue posible en los dos casos gracias al interés y disponibilidad de los sujetos al compartir colectivamente su sentir. En el caso de la AG eso fue posible al observar que los sujetos ya expresaban sus emociones en otros espacios colectivos como las asambleas semanales. En el caso del CS dio lugar a una reflexión del colectivo acerca de la relevancia de la dimensión emocional en su lucha que sigue al día de hoy. En el caso del CS, el trabajo de campo se llevó a cabo a partir de mayo de 2015, mientras que en agosto de 2017 empezó el seguimiento de la lucha de la AG en la Ciudad de México.

\section{RESULTADOS}

Para poder mostrar el papel de las emociones en la defensa del medioambiente, se ha resumido en la Figura 1 las emociones más relevantes que caracterizan las dos luchas, las cuales se vinculan a los valores biosféricos que caracterizan a estos colectivos. La Figura 1, no pretende mostrar todas las emociones que se pueden observar en estas experiencias, pero sí evidenciar aquellas que son centrales para 
comprender lo que mobiliza a estos sujetos a

defender el medioambiente.

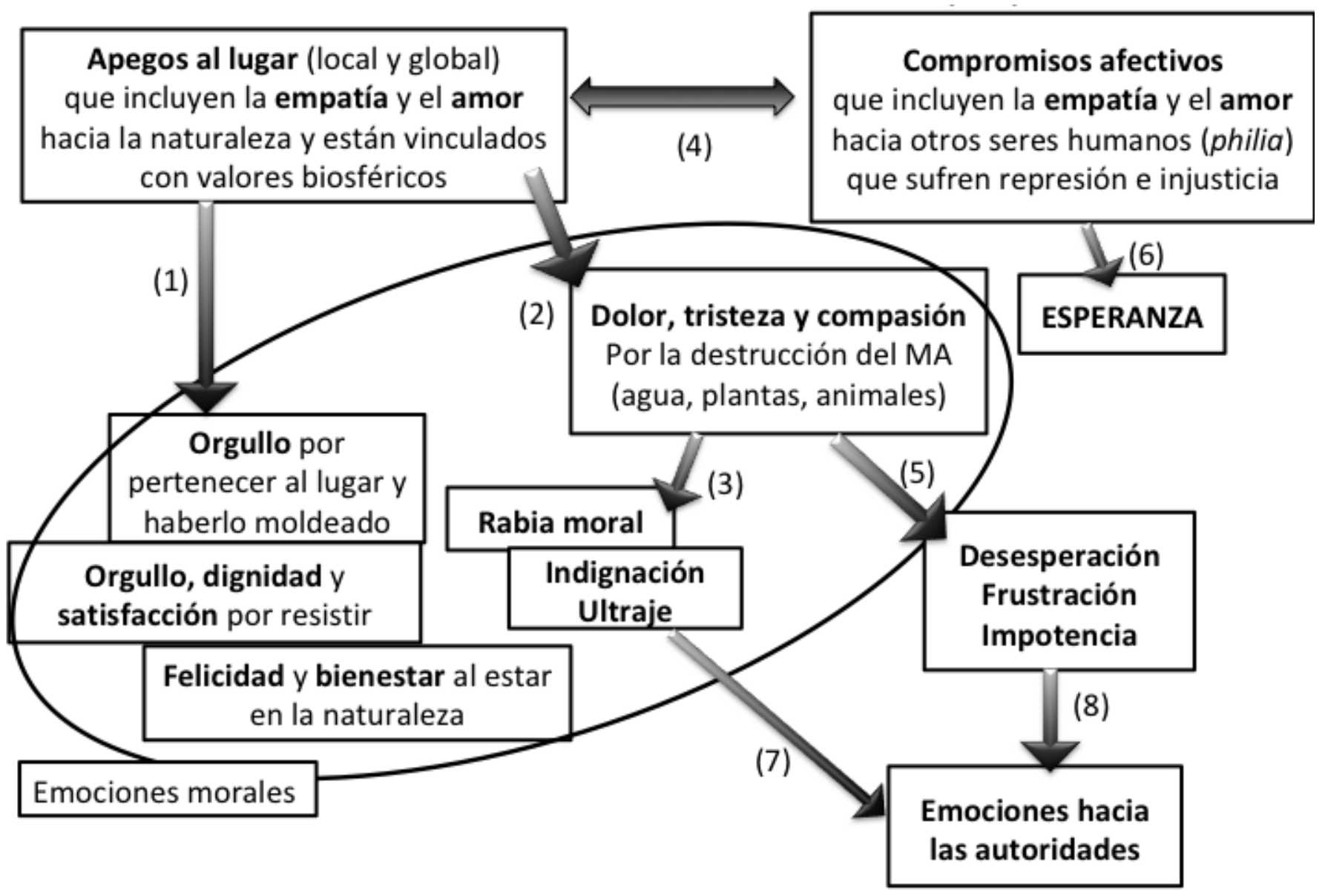

Figura 1. La dimensión emocional de la defensa del medio ambiente (MA).

La Figura 1 sugiere que los vínculos afectivos hacia las personas y los lugares son factores explicativos de estas experiencias. Mientras que los compromisos entre personas están presentes en todas las experiencias de lucha, el apego al lugar, a nivel local y global, es caraterístico de los conflictos socioambientales.

El análisis de los dos casos de estudio sugiere que en la categoría de los compromisos afectivos, el amor hacia las personas y la naturaleza juega un rol central en la defensa del medioambiente, junto con la empatía. En el campo de estudio de los movimientos sociales, el amor como atracción erótica entre participantes ha sido identificado como un disruptor (Goodwin, 1997), mientras se identifica como aglutinador el amor que sienten entre ellas las mujeres que luchan en movimientos de víctimas (Bayard de Volo, 2006) y feministas (Taylor, 1989). Sin embargo, no se han encontrado referencias que analicen el amor 
hacia la naturaleza como un factor explicativo de la defensa del medioambiente.

A continuación, se identificarán las relaciones de la Figura 1 con los números que se refieren a las flechas. Los números solo sirven para identificar la relación y no indican procesos consecutivos. Los apegos al lugar local y global, que incluyen amor y empatía hacia los elementos naturales, generan emociones morales como el orgullo por pertenecer a un lugar, el orgullo, la dignidad y la satisfacción por defenderlo, la felicidad y el bienestar al experimentar el vínculo con la naturaleza (ver flecha 1, Figura 1), pero también el dolor, la tristeza y la compasión al verlo destruido (ver flecha 2, Figura 1).

Ese dolor, como veremos a continuación, genera rabia moral, indignación y ultraje (ver flecha 3 , Figura 1), que a su vez generan emociones como la desconfianza o el desprecio hacia las autoridades que destruyen la naturaleza (ver flecha 7, Figura 1). El dolor por la destrucción del medioambiente, que como veremos es un indicador del amor hacia el mismo, genera desesperación, frustración e impotencia al no poder frenar la destrucción ambiental (ver flecha 5, Figura 1). Estas emociones, por un lado, alimentan las emociones hacia las autoridades (ver flecha 8, Figura 1), pero por otro pueden llevar al agotamiento o a la desmovilización, si no son manejadas colectivamente (Gravante \& Poma, 2018). También pude observar que en las dos experiencias los compromisos afectivos hacia miembros del colectivo $u$ otros que sufren violencia e injusticia, generan esperanza (ver flecha 6, Figura 1). Pero también, estos compromisos afectivos están vinculados con los apegos al lugar, siendo el territorio no sólo el espacio físico, sino también el conjunto de relaciones sociales con sus habitantes (ver flecha 4, Figura 1).

Las emociones como factores explicativos de la defensa del medio ambiente

Un elemento clave para comprender los conflictos socioambientales son los apegos al lugar, a nivel local y global. Ese vínculo ha sido definido como "la relación simbólica formada por personas que otorgan significados emocionales/afectivos culturalmente compartidos a un particular espacio o terreno que proporciona la base para la comprensión y la relación con el medio ambiente del grupo y del individuo" (Low, 1992, p. 165). A nivel local, el apego al lugar facilita la defensa del territorio y la oposición contra aquellas instalaciones que los habitantes perciben como amenazadoras hacia su territorio (Devine-Wright, 2009; Poma, 2017), mientras que a nivel global el apego se ha relacionado con comportamientos proambientales $y$ el compromiso hacia el medioambiente (Devine-Wright \& Batel, 2017). $\mathrm{El}$ apego al lugar local influye en la defensa del territorio al generar emociones morales como el orgullo por pertecer al lugar (ver flecha 1, Figura 1), como se puede observar en los siguientes testimonios:

"Traemos esta emoción de ver limpio nuestro espacio por nuestras propias manos. Es un orgullo". (Mujer adulta de la AG)

"¿Cuánta gente en la ciudad ha visto como yo he visto los animalitos del cerro? Nadie. 
Porque estamos resistiendo aquí y tenemos derecho de ver esto. Allí entra el orgullo". (Mujer adulta del CS).

$\mathrm{Al}$ defender el territorio que se ama y al que las personas sienten pertenecer, se generan además otras emociones como el orgullo, la dignidad o la satisfacción para no quedarse con las manos cruzadas frente a la devastación ambiental. Estas emociones son también fortalecidas por el apego al lugar global, es decir, hacia la naturaza. El apego al lugar global se observa en la forma en que los integrantes de los dos colectivos se refieren a los recursos naturales como a seres vivos, que sufren, en el caso de los árboles talados (CS), o como alguien que está resistiendo y grita, en el caso del manantial (AG). Eso explica el uso del concepto "ecocidio" para describir la devastación del territorio. Considerar la naturaleza como un ser vivo es una característica de algunos pueblos indígenas, que emerge en diferentes movimientos y los casos analizados en esta investigación, cuyos integrantes, aunque no vivan en comunidades indígenas, han sido influenciados por diferentes culturas indígenas. De la misma manera que el lema de los Lakota Mni Wicomi ("el agua es vida") en el movimiento NODAPL en Estados Unidos (Streeby, 2018), la AG reinvindica que "el agua es vida y la vida se defiende", mientras que los miembros del CS hacen lo propio al señalar que el bosque está vivo. Reconocer la personalidad del agua y las plantas, desafía el antropocentrismo de Occidente que otorga personalidad sólo a los humanos (Valandra, 2016), y se puede remontar a las raíces culturales que algunos integrantes de estos colectivos tienen, como pude observar en el altar que crearon en el plantón de la AG y en sus rituales para agradecer a la madre tierra, así como en las experiencias biográficas compartidas en las entrevistas:

"Traemos algún tipo de emociones, ideas, ya muy cercanas porque somos de Mezcala, mi familia es de Mezcala. Una relación muy cercana con la tierra todavía, creo que también eso tiene que ver con ahora reapropiarnos del territorio, porque eso es lo que hemos hecho, reapropiarnos del territorio". (Hombre adulto del CS).

Esta vinculación con la naturaleza, que fortalece los valores biosféricos de estos sujetos, es posible gracias a la empatía hacia los animales y los elementos naturales (agua y árboles) amenazados, que conduce a los miembros de la AG y del CS a sentir compasión (ver flecha 2, Figura 1) por aquellos. Como muestra Jasper (2018), la compasión entrega sentido a la vida de otras especies desde un punto de vista subjetivo y en su propia dignidad. No obstante, en la literatura sobre movimientos sociales la compasión hacia los seres no-humanos sólo se ha analizado en movimientos animalistas.

En los casos analizados, se observó que tal como para los animalistas anarcopunk mexicanos, cuya empatía "surge de una condición compartida como animales de uso, desuso y de carga" (frase presente en un cartel del movimiento anarkopunk mexicano, rescatada de un archivo fotográfico privado) la empatía que caracteriza a la AG y al CS nace de sentir el manantial y el bosque como un ser más, que al igual que ellos pertenece a su territorio y está amenazado por el desarrollo inmobiliario. Esta 
conexión entre habitantes amenazados, humanos y no humanos, se observa en el CS al considerar a los animales del bosque como víctimas del ecocidio y símbolo de la lucha, y en el caso de la AG, a convertir unos patos que solían visitar el manantial en símbolo de su lucha. La desaparición de los patos adquiere significado si se considera que estos desarrollos inmobiliarios están pensados para personas de clases sociales más elevadas, que podrían llevar en el futuro a un desplazamiento de estas familias de su territorio, de la misma manera como ha pasado con los patos. En estos casos, la empatía hacia los animales y los elementos naturales se construye a partir de sentir que las otras especies son exactamente como ellos, porque también son víctimas del modelo de desarrollo capitalista. Este resultado se confirma si consideramos que una metáfora comúnmente usada por los afectados ambientales en México es la de ser tratados como animales por parte de las autoridades (Poma, 2017).

Además de la motivación para la acción, los apegos al lugar permiten comprender también las estrategias que estos colectivos llevan a cabo. La elección de ocupar temporalmente la avenida (AG) y el bosque (CS), de marchar y detener las maquinarias usando sus propios cuerpos, entre otras acciones, tienen el objetivo de detener la destrucción del acuífero y del bosque, respetando así los lugares y sus habitantes. El vínculo que une a estas personas con sus lugares (ver flecha 4, Figura 1) hace que ellos quieran, antes que nada, permanecer y vivir en él, por lo que las acciones directas no violentas que llevan a cabo, de manera prefigurativa, están dirigidas a construir comunidad y transmitir conocimiento y amor hacia el territorio.

A pesar de que estas luchas pueden frenar la destrucción ambiental, pero sin alcanzar a pararla -elemento que como afirma Jasper (2011) es central en todo movimiento-, el uso de la violencia no está contemplado. Ello, no sólo porque produciría más represión, poniendo en riesgo a los seres queridos de los activistas, sino también porque implicaría romper o cambiar la relación con el territorio y sus habitantes. El fin de defender la vida, a través de la oposión a la destrucción del manantial y el bosque, es así perseguido, de manera prefigurativa, con medios que defienden y reproducen la vida, humana y no humana.

Como se observa en la Figura 1, los compromisos afectivos entre personas evocan además un sentimiento colectivo de esperanza (ver flecha 6 , Figura 1), como lo definió una mujer de la AG en un grupo focal. La esperanza se alimenta en estas experiencias del amor como lazos filiales, es decir la "relación humana basada en el cariño y afecto mutuos" (Chabot, 2008, p. 811). Conviviendo con miembros de una misma familia en estos colectivos, los lazos filiales hacia hijos, sobrinos y nietos juegan un rol importante para evocar esperanza. Como afirmó una integrante del CS hablando de su sobrina:

"[Ella] es como el arbolito que sembramos en el bosque, que sabemos que puede ser que no lo vamos a ver así de grande, pero que alguien lo volverá a ver. [Ella] es la esperanza viva.[...] Tengo la esperanza que le cuente a todo el mundo que había aquí gente, un bosque, etc. Esa chiquita armando una 
revolución, chiquita como la nuestra”. (Mujer adulta del CS).

El compromiso con las nuevas generaciones es central en los conflictos socioambientales, porque proporciona esperanza. Al involucrar a los niños en la defensa del agua y el bosque, las luchas se convierten en espacios de aprendizaje y resistencia, donde la dignidad de no rendirse se transforma en un ejemplo a seguir. La importancia de la educación y la experiencia en la infancia emergió también en el trabajo de campo, al entrevistar a jóvenes de los dos colectivos, que expresaron cómo sus valores biosféricos son el resultado de su experiencia personal:

"El bosque tiene muchos significados, yo crecí aquí [...] para mí entonces fue una vivencia muy cercana desde pequeño. Estar aquí en el bosque [...] a mí eso me marcó, fue algo muy cercano desde la infancia". (Hombre adulto del CS).

"Muchos años fui scout, entonces este contexto social me permitió poco a poco acercarme a comprender el proceso del agua”. (Hombre joven de la AG).

Las acciones que llevan a cabo los integrantes del CS (e.g., reforestación, limpieza del bosque, los viveros de plantas, los campamentos de verano para niños), no sólo sirven para evitar la destrucción del bosque, sino también para trasmitir a las nuevas generaciones dignidad y respeto a la vida, así como el amor y la belleza del bosque, para que en el futuro puedan defenderlos ellos también. Las estrategias de los colectivos están así vinculadas con una visión de largo plazo, ya que estos sujetos son conscientes de que la destrucción del medioambiente va a seguir, y por eso el aquí y ahora es clave para generar las condiciones para que haya resistencia en el futuro.

En lo que concierne a la AG, esa visión a futuro, que de manera prefigurativa empieza en el aquí y ahora, se materializa en la Escuela Popular Piedra y Manantial. Este espacio de autoaprendizaje genera muchos beneficios emocionales, como difundir la esperanza y superar la impotencia, gracias a la vinculación con otras experiencias de lucha. Además, proporciona a los miembros de la AG autoestima, por haberse convertido en referentes de muchos movimientos, y por recibir señales de admiración por parte de quienes participan en la Escuela. El análisis de los vínculos afectivos hacia personas y lugares muestra que también en los conflictos socioambientales el amor "es una fuerza para la inclusión social, la transformación y la justicia" (Chabot, 2008, p. 808). Sin embargo, aunque la presencia de diferentes tipos de amor en los casos analizados pueda ser evidente, el estudio de la dimensión emocional trata de explicar también cómo estos tipos de amor interactúan con otras emociones. En los casos analizados, un vínculo que resulta central es la relación entre el amor por la naturaleza y el dolor y la tristeza que sienten los integrantes de los colectivos al verla destruida (ver flecha 2 , Figura $1)$.

Lo que emergió en el trabajo de campo es que el amor que los entrevistados sienten hacia el entorno es directamente proporcional al dolor y la tristeza que sienten por su destrucción. Estas emociones son morales en cuanto dependen del 
significado que los sujetos atribuyen al agua y al bosque, y de sus valores biosféricos. Comprender ese dolor es central para comprender el vínculo que estos sujetos tienen con la naturaleza, y el compromiso con la resistencia. Comprender la relación entre el amor y el dolor, en estos casos, permite también superar una de las dificultades en las que incurrimos los que analizamos la dimensión emocional de la protesta: cómo verificar la presencia de ciertas emociones que emergen en el trabajo de campo (ya sea a través de la observación participante o en las entrevistas). El dolor generado por la destrucción del acuífero y del bosque se convierte así en un indicador de la intensidad del apego al lugar de estos sujetos.

El dolor generado por la destrucción ambiental es una emoción que los integrantes de los colectivos comparten entre ellos. Por ejemplo, durante una discusión colectiva con integrantes de la AG, una participante compartió el dolor que sintió al escuchar el agua que la empresa constructora estaba tirando en el desagüe durante la noche, mientras ella estaba de guardia. Ella afirmó que al escuchar este ruido se puso a llorar, y que en ese momento sintió que el manantial estaba vivo y sufriendo. El compartir este dolor con los demás hace que se fortalezca el vínculo entre ellos, ya que como dijo una mujer mayor de la AG, "sabemos que están los proyectos de muerte, pero sentirlos, vivirlos, es diferente". Las emociones permiten así que se experimente el impacto de la destrucción de manera visceral, lo cual influye en la legitimación de la lucha y en la motivación para la acción. Por esta razón, el dolor es una emoción legítima y expresada públicamente como emoción motivadora de la acción, como muestra este extracto de la convocatoria de la asamblea especial para recordar el primer aniversario del desalojo del primer plantón, el 5 de diciembre de 2017:

"Es cierto, nos lastima ver cómo avanza el concreto hacia el cielo y escuchar cómo nuestro manantial día y noche nos grita que no permitamos más este ecocidio, pero nuestro dolor se transforma en organización, se convierte en aprendizaje”. Los sueños no se desalojan, AG, archivo particular.

Sin embargo, el dolor puede también generar otras emociones como la desesperación, la frustración y la impotencia (ver flecha 5, Figura 1) al no poder defender el agua o el bosque, y que a su vez influyen en las emociones hacia las autoridades. Como muestra el siguente testimonio, el dolor por la tala de los árboles genera al mismo tiempo impotencia y alimenta el ultraje hacia las autoridades (ver flecha 7, Figura 1):

"Ellos vienen y con sus máquinas, con una facilidad tumban el trabajo o árboles que tienen cientos de años y con una facilidad los echan al suelo y los trituran [Llora] Sí, sientes impotencia, porque los gobiernos son sordos, y nosotros ya lo sabemos". (Mujer adulta del $\mathrm{CS}$ ).

Emociones como la desesperación, la frustración y la impotencia generadas por la destrucción del medioambiente (ver flecha 5, Figura 1), conducen a ambos grupos a identificar a las autoridades como "cínicas" y "sin corazón", porque como sostuvo una mujer adulta de la $\mathrm{AG}$, 
"al gobierno no le importa, es insensible a todo esto". Es así que se definen y fortalecen las emociones hacia las autoridades (ver flecha 8, Figura 1), que influyen en el rechazo de estos colectivos a colaborar con partidos y participar en la política “convencional". El dolor también tiene que ser manejado para que no lleve al agotamiento o estados de ánimo como la depresión. Estos colectivos han así desarrollado estrategias de manejo emocional para poder sobrellevar el dolor colectivamente a través de la organización de actividades lúdicas y festivas, que permiten además fortalecer las relaciones entre los miembros.

Actividades que comprendan música, comida, teatro, así como la reforestación llevada a cabo por el CS o la milpa plantada en el camellón de la avenida por parte de la AG, junto con talleres de herbolaria (AG) o la limpieza del bosque (CS), son todas actividades que además de fortalecer los vínculos con el territorio y los participantes, permiten sobrellevar el dolor y generan bienestar y satisfacción al estar haciendo acciones que son coherentes con sus valores biosféricos. El dolor puede también ser trasformado en rabia y acción por parte de los integrantes de un colectivo. La rabia es una emoción que se ha considerado en el estudio de los movimientos sociales por su capacidad de incentivar la protesta (Holmes, 2004; Gould, 2009; Jasper, 2018). Esta rabia en la que se canaliza el dolor generado por la destrucción del medioambiente es una rabia moral (Jasper, 2012,2018 ), que se vincula con otras emociones morales como la indignación y el ultraje, como se muestra en Figura 1, flecha 3, y como se puede observar en las palabras de la AG, que van a concluir este apartado:

"Nuestros patos ¿qué le hicieron a nuestros patos?, ¿dónde está su andar que alegraba a nuestro manantial?, digan [la inmobiliaria y el gobierno] ¿en qué parte de su conciencia guardaron la imagen de las mejillas brillantes de llanto, de las trenzas mojadas de llanto provocadas por tanta injusticia? Pero el coraje [ira] se levantó y se hizo fuerza, se hizo y hace organización, se teje diariamente con cada una y uno de nosotros, por eso seguimos aquí, construyendo". Los sueños no se desalojan, AG (2017), archivo particular.

\section{DISCUSIÓN Y CONCLUSIONES}

Apoyándose en Jasper (1997, 2018), quien evidencia la relevancia del vínculo afectivo con el territorio en los conflictos socio ambientales, así como Devine-Wright (2009), Devine-Wright y Batel (2017) y Devine-Wright, Price y Leviston (2015), quienes muestran el papel de los apegos al lugar en la oposición a obras indeseadas, el presente artículo se propuso mostrar cómo los apegos al lugar, al vincularese con otras emociones, motivan la defensa del medio ambiente en el contexto urbano.

En consonancia con Jasper (2018) quien plantea que "los habitantes de las ciudades sienten un apego más abstracto a la tierra amenazada, 
frecuentemente basada

en valores ambientalistas" (p. 94), esta investigación mostró que los defensores del territorio sufren el escaso apego al lugar, y consecuente compromiso con el medio ambiente, de sus vecinos (Poma \& Gravante 2015, p. 104). Sin embargo, los casos analizados muestran que en México, en áreas urbanas, existen sujetos con valores biosféricos y fuerte apegos al lugar, a nivel local y global, derivados de su experiencia en el territorio y la resignificación de la naturaleza de la que se sienten parte, que a su vez explican sus valores biosféricos.

La interacción entre los apegos al lugar que sienten estos sujetos, sus valores biosféricos y las emociones morales, permite comprender su compromiso en defender el medioambiente. Así, se mostró que estos sujetos sienten un profundo dolor frente a la destrucción del medioambiente, que es expresado explícitamente tanto en las entrevistas individuales como en el discurso publico de los colectivos. El dolor por la pérdida de seres queridos se vuelve movilizador, como mostró también Gould (2009), al ser canalizado en otras emociones morales como la rabia, la indigarción o el ultraje.

El dolor, además de ser movilizador, se convierte en un indicador de los apegos al lugar y el amor hacia la naturaleza. Si bien aún falta trabajo para comprender el papel de diferentes tipos de amor y cómo estos interactúan con otras emociones, los casos analizados resaltan la importancia de diferentes tipos de amor, filial y hacia la naturaleza, en la defensa del medioambiente.
Finalmente, el análisis muestra también cómo el carácter prefigurativo de estas luchas está ligado a los apegos al lugar por un lado y a los compromisos afectivos por el otro. Estos resultados evidencian la relación entre las emociones y las tácticas prefigurativas de los colectivos, elemento escasamente analizado en la literatura. El análisis de los dos casos evidencia así la relevancia de comprender en contextos específicos la interacción entre diferentes emociones y su impacto en los conflictos socioambientales.

La comparación a su vez permitió identificar patrones en la interacción entre emociones, lo que ofrece un entendimiento de la dimensión emocional de los conflictos socio ambientales. De hecho, es interesante destacar que pese a las diferencias entre las dos luchas estudiadas (duración, geografía y elemento natural amenazado), las emociones analizadas emergieron sin diferencias relevantes, y fueron sentidas por todos los miembros, independientemente del género y la edad.

Siendo las emociones constructos socioculturales cuyo manejo y expresión dependen de la subcultura de los grupos, de su ideología y de la cultura del país al que pertenecen, son necesarios más estudios para poder comprobar si la interacción observada en los casos analizados ocurre en otros contextos. Nuevos trabajos empíricos son así necesarios para fortalecer esta teoria de la acción y ayudar a comprender mejor lo que moviliza a las personas a defender el medioambiente.

Por otra parte, la apropiación y aplicación de estos resultados por parte de los colectivos en 
Poma, A. (2019). El papel de las emociones en la defensa del medioambiente: Un enfoque sociológico. Revista de Sociología, 34(1), 43-60. doi: 10.5354/0719-529X.2019.54269

defensa del medioambiente, podría contribuir a promover mayor activismo ambiental, por ejemplo, diseñando campañas o proyectos para fomentar el apego al lugar, tanto local como global, en poblaciones de distintas geografías y culturas. Como escribió Hochschild (2008), el capitalismo no es sólo un modelo económico, sino también cultural, que promueve ciertas reglas del sentir. Para superar la crisis ambiental global es necesario desarrollar así una nueva cultura, también emocional, en la que amar el planeta y a sus habitantes; sufrir cada vez que se cometa un daño ambiental; y admirar a quienes defienden el medio ambiente, se conviertan en nuevas reglas del sentir.

\section{REFERENCIAS}

Bayard de Volo, L. (2006). The dynamics of emotion and activism: Grief, gender, and collective identity in revolutionary Nicaragua. Mobilization, 11(4), 461-474.

Chabot, S. (2008). Love and revolution. Critical Sociology, 34(6), 803-828. doi: $10.1177 / 0896920508095100$

Dear, M. (1992). Understanding and overcoming the NIMBY sindrome. Journal of the American Planning Association, 58(3), 288301. doi: 10.1080/01944369208975808.

Devine-Wright, P. (2009). Rethinking NIMBYism: The role of place attachment and place identity in explaining placeprotective action. Journal of Community \& Applied Social Psychology, 19(6), 426-441. doi: 10.1002/casp.1004.

Devine-Wright, P. \& Batel, S. (2017). My neighbourhood, my country or my planet? The influence of multiple place attachments and climate change concern on social acceptance of energy infrastructure. Global Environmental Change, 47(1), 110-120. doi: 10.1016/j.gloenvcha.2017.08.003.

Devine-Wright, P., Price J., \& Leviston, Z. (2015). My country or my planet? Exploring the influence of multiple place attachments and ideological beliefs upon climate change attitudes and opinions. Global
Environmental Change, 30(1), 68-79. doi: 10.1016/j.gloenvcha.2014.10.012.

Diani, M. (2015). Revisando el concepto de movimiento social. Encrucijadas. Revista Crítica de Ciencias Sociales, $9(1), 1-16$.

Enciso, A. (2016). Hay en México 420 conflictos socioambientales: Investigador. La Jornada, Disponible https://www.jornada.com.mx/2016/02/10/soc iedad/038n1soc.

Epstein, B. (1991). Political protest and cultural revolution: Nonviolent direct action in the 1970s and 1980s. Berkeley, CA: University of California Press.

Flam, H. (2000). The emotional 'Man' and the problem of collective action. Berlin: Peter Lang.

Flam, H. \& King, D. (2005). Emotions and social movements. London/New York: Routledge.

Goodwin, J. (1997). The libidinal constitution of a high-risk social movement: Affectual ties and solidarity in the huk rebellion. American Sociological Review, 62(1), 53-69.

Goodwin, J., Jasper J. \& Polletta P. (Eds.). (2001). Passionate politics: Emotions and social movements. Chicago, IL: University of Chicago Press.

Goodwin, J. \& Pfaff, S. (2001). Emotion work in high-risk social movements: Managing 
Poma, A. (2019). El papel de las emociones en la defensa del medioambiente: Un enfoque sociológico. Revista de Sociología, 34(1), 43-60. doi: 10.5354/0719-529X.2019.54269

Fifear in the U.S. and East German Civil Rights Movements. En: J. Goodwin, J. Jasper, \& F. Polletta (Eds.), Passionate politics: Emotions and social movements (pp. 282-302). Chicago, IL: University of Chicago Press.

Gould, D. (2009). Moving politics: Emotion and $A C T$ UP's fight against AIDS. Chicago, IL: University of Chicago Press.

Graeber, D. (2009). Direct action: An ethnography. Oakland, CA: AK Press.

Gravante, T. \& Poma, A. (2018). Manejo emocional y acción colectiva: Las emociones en la arena de la lucha política. Estudios Sociológicos, 36(108), 593-616. doi: 10.24201/ES.2018V36N108.1612.

Hochschild, A. (1979). Emotion work, feeling rules, and social structure. American Journal of Sociology, 85(3), 551-575. doi: $10.1086 / 227049$

Hochschild, A. (1983). The managed heart: the commercialization of human feeling. Berkely, CA: University of California Press.

Hochschild, A. (2008). La mercantilización de la vida íntima. Buenos Aires: Katz Editores.

Holmes, M. (2004). Feeling beyond rules. politicizing the sociology of emotion and anger in feminist politics. European Journal of Social Theory, $7(2), 209-227$. doi: 10.1177/1368431004041752.

Jasper, J. (1997). The art moral of protest: culture, biography, and creativity in social movements. Chicago: University of Chicago Press.

Jasper, J. (1998). The emotions of protest: Affective and reactive emotions in and around social movements. Sociological Forum, 13(3), 397-421. doi: 10.1023/A:1022175308081

Jasper, J. (2012). Las emociones y los movimientos sociales: veinte años de teoría e investigación. Revista Latinoamericana de Estudios sobre Cuerpos, Emociones y Sociedad, 4(10), 46-66.
Jasper, J. (2014). Protest: A cultural introduction to social movements. Cambridge, UK: Policy Press.

Jasper, J. (2018). The Emotions of protest. Chicago: University of Chicago Press.

Johnston, H. \& Klandermans, B. (Eds.). (1995). Social movements and culture. Minneapolis, MN: University of Minnesota Press.

Kleres J. \& Wettergren Å. (2017). Fear, hope, anger, and guilt in climate activism. Social Movement Studies, 16(5), 507-519. doi: 10.1080/14742837.2017.1344546

Leff, E. (2004). Racionalidad ambiental. La reapropiación social de la naturaleza. Ciudad de México: Siglo XXI.

Leff, E. (2011). Sustentabilidad y racionalidad ambiental: hacia "otro" programa de sociología ambiental. Revista Mexicana de Sociología, 73(1), 5-46. doi: 10.22201/iis.01882503p.2011.1.23562

Low, S. (1992). Symbolic ties that bind. place attachment in the plaza. En S. Low \& I. Altman (Eds.), Place Attachment (pp. 165185). New York: Plenum.

Maeckelbergh, M. (2011). Doing is believing: Prefiguration as strategic practice in the alterglobalization movement. Social Movement Studies, 10(1), 1-20. doi: 10.1080/14742837.2011.545223.

Martínez Alier, J. (2004). El ecologismo de los pobres. Conflictos ambientales y lenguajes de valoración. Barcelona: Icaria.

Pearlman, W. (2013). Emotions and the microfoundations of the Arab Uprisings. Perspectives on Politics, 11(2), 307-409. doi: 10.1017/S1537592713001072.

Perlaviciute, G., Steg, L., Contzen, N., Roeser S., \& Huijts N. (2018). Emotional responses to energy projects: Insights for responsible decision making in a sustainable energy transition. Sustainability, 10(7) 2526, 1-12. doi: 10.3390/su10072526.

Poma, A. (2014). Emociones y subjetividad. Un análisis desde abajo de las luchas por la 
Poma, A. (2019). El papel de las emociones en la defensa del medioambiente: Un enfoque sociológico. Revista de Sociología, 34(1), 43-60. doi: 10.5354/0719-529X.2019.54269

defensa del territorio. Papers, 99(3), 377401. doi: 10.5565/rev/papers.602

Poma, A. (2017). Defendiendo territorio y dignidad. Emociones y cambio cultural en luchas contra represas en España y México. Campina Grande, Paraíba, Brasil: Universidade Estatal de Paraiba e ITESO.

Poma, A. (2019). Cambio climático y activismo ambiental: El papel de los apegos al lugar. Tla-melaua, Revista de Ciencias Sociales, 12(46), 212-237. doi: 10.32399/rtla.0.46.573

Poma, A. \& Gravante, T. (Eds.). (2015). Resistencias y autogestión en contra del despojo del agua y del territorio en la Zona Metropolitana de Guadalajara: logros y retos. Waterlat-Gobacit, Network Working Papers, 2(18).

Poma, A. \& Gravante, T. (2017a). Emociones, protesta y acción colectiva: Estado del arte y avances. Aposta. Revista de Ciencias Sociales, 74(1), 31-62.

Poma, A. \& Gravante, T. (2017b). Emotions in inter-Action in environmental resistances. The case of Comité Salvabosque in Mexico. Partecipacione e Conflitto, 10(3), 896-925. doi: 10.1285/i20356609v10i3p896

Poma, A. \& Gravante, T. (2018). Emociones, identidad colectiva y estrategias en los conflictos socio-ambientales. Andamios.
Revista de Investigación Social, 15(36), 287309. doi: 10.29092/uacm.v15i36.611.

Steg, L. (2016). Values, norms, and intrinsic motivation to act proenvironmentally. Annual Review of Environment and Resources, 41, 277-92. doi: 10.1146/annurev-environ-110615-085947.

Streeby, S. (2018). Imagining the future of climate change. World-making through science fiction and activism. Oackland, CA: University of Califonia Press.

Taylor, V. (1989). Social movement continuity: The women's movement in abeyance. American Sociological Review, 54(5), 761775. doi: 10.2307/2117752.

Valandra, E. (2016). We Are Blood Relatives: No to the DAPL. Hot Spots: Society for Cultural Antrhropology. Disponible en https://culanth.org/fieldsights/we-are-bloodrelatives-no-to-the-dapl

Yates, L. (2014). Rethinking prefiguration: Alternatives, micropolitics and goals in social movements. Social Movement Studies, 14(1), 1-21. doi: 10.1080/14742837.2013.870883

Manuscrito recibido: 06-05-2019 Manuscrito aceptado: 05-08-2019 